\title{
Resiliencia, Personalidad Resistente y Crecimiento en Cuidadores de Personas con Demencia en el Entorno Familiar: Una Revisión
}

\section{Resilience, Hardiness and Growth in Dementia Patients' Family Caregivers: A Review}

\author{
Violeta Fernández-Lansac y María Crespo L. \\ Universidad Complutense de Madrid, España
}

\begin{abstract}
Resumen. La investigación en torno al cuidado no profesional de mayores con demencia ha proliferado a lo largo de los últimos años, constatando muchos autores que, del mismo modo en que el cuidado se asocia a un buen número de consecuencias negativas, diversos efectos positivos pueden derivarse de esta experiencia. Sin embargo, y a pesar del impacto de la corriente positiva en la actualidad, son muy escasos los estudios que valoran la personalidad resistente (hardiness), la resiliencia y el crecimiento personal en cuidadores. El presente artículo hace una revisión sistemática de las contribuciones más relevantes a este campo, a partir del análisis de nueve publicaciones. Todos estos trabajos evalúan, de un modo cuantitativo, la resiliencia, el hardiness o el crecimiento en familiares de personas con demencia. Las características y resultados de dichos trabajos serán valoradas, teniendo en consideración sus implicaciones para el estudio y la atención a los cuidadores.
\end{abstract}

Palabras clave: resiliencia, personalidad resistente, crecimiento, cuidador, demencia.

\begin{abstract}
In the last few years, research into non professional caregiving for dementia patients has increased, and has shown that such caregiving carries both negative and positive effects. Nevertheless, in spite of the growing influence of positive psychology, studies focused on resilience, hardiness and personal growth in caregivers remain fairly scarce. The present article aimed to systematically review the existing studies on this topic, analyzing nine studies that assess resilience, hardiness or growth in dementia patients' family caregivers. The features and results of these studies will be discussed, considering their implications for the study of, and attention to, caregivers.

Keywords: resilience, hardiness, growth, caregiver, dementia.
\end{abstract}

\section{Introducción}

En los últimos años el cuidado no profesional de personas mayores dependientes se ha convertido en un tema de especial relevancia, tanto desde un punto de vista social como desde un punto de vista clínico. Debido esencialmente al envejecimiento poblacio-

La correspondencia sobre este artículo debe enviarse a María Crespo L. al e-mail:mcrespo@psi.ucm.es nal y al incremento de enfermedades degenerativas, cada vez hay un mayor número de cuidadores y éstos, además, ocupan esa posición durante periodos más prolongados.

La investigación realizada en las tres últimas décadas ha evidenciado de manera consistente que los cuidadores no profesionales (en su mayoría familiares) de ancianos están, por el hecho de serlo, sometidos a una situación de estrés crónico con importantes repercusiones en su estado físico y emo- 
cional. De este modo, se han registrado dentro de este grupo, en comparación con la población general, elevados índices de ira, sintomatología ansiosa y depresiva, menores niveles de bienestar subjetivo y autoeficacia (e.g. Pinquart y Sörensen, 2003a; Schulz, O’Brien, Bookwala y Fleissner, 1995), así como una mayor probabilidad de sufrir alteraciones en su sistema cardiovascular e inmune, como consecuencia del impacto del estrés al que se somete diariamente (Vitaliano, Schulz, Kiecolt-Glaser y Grant, 1997). Además algunos estudios apuntan a que el estrés del cuidador se verá incrementado notablemente en caso de que el mayor al que asista padezca algún tipo de demencia (Pinquart y Sörensen, 2003a).

No obstante, recientemente distintos autores han advertido que, del mismo modo en que el cuidado constituye una notable fuente de estrés, un buen número de consecuencias positivas pueden derivarse de tal experiencia. La posibilidad de auxiliar a una persona que lo necesita (más aún si se la quiere), e incluso la posibilidad de acompañarla en los últimos momentos de su vida, puede ser percibida por el cuidador como una oportunidad para su propio desarrollo personal y traducirse, así, en sentimientos de bienestar y satisfacción. En esta línea, Cohen, Colantonio y Vernich (2002) han encontrado que en torno al $73 \%$ de los cuidadores no profesionales de ancianos señalan al menos un aspecto positivo de esta experiencia, lo cual además es indicativo de menores niveles de carga percibida y mejores autoevaluaciones con respecto a su salud. Las mayores fuentes de satisfacción que identifican los cuidadores están relacionadas con la sensación de compañía, de utilidad y de estar cumpliendo con su deber u obligación. Asimismo los cuidadores también refieren momentos placenteros o divertidos durante el desempeño de su labor (Cohen et al., 2002)

En general, todos estos aspectos positivos son englobados bajo el término beneficios del cuidado (uplifts), concepto que abarca además sentimientos tales como los relativos al aprecio por la cercanía con el receptor de cuidados o al orgullo derivado de la propia capacidad para afrontar las crisis (Kramer, 1997). Según los datos arrojados por el meta-análisis de Pinquart y Sörensen (2003b), que puede con- siderarse el más exhaustivo sobre el tema, y en el que se identifican 228 estudios que valoran el efecto de diversos aspectos positivos del cuidado (en concreto, satisfacción, aspectos placenteros o ganancias percibidas), dichos beneficios se asocian con menores niveles de carga y depresión, amortiguando, presumiblemente, el efecto negativo de la situación de asistencia sobre el estado emocional del cuidador. Sin embargo, y dada la naturaleza correlacional de los datos obtenidos, cabe la posibilidad de efectuar interpretaciones alternativas, tales como que los cuidadores con mayor malestar emocional puedan percibir en menor medida los aspectos positivos asociados al cuidado. Además, los resultados de Pinquart y Sörensen (2003b) evidencian la ausencia de relación entre la percepción de beneficios del cuidado y los estresores objetivos de la situación (e.g. tareas, horas dedicadas al cuidado), lo cual apunta a que la percepción de beneficios depende de otros aspectos de la relación cuidador-receptor de cuidados, aspectos entre los que se incluyen la cercanía mutua, la motivación o características de la propia personalidad del cuidador. Adicionalmente, y a nivel contextual, diversos investigadores enfatizan el papel que ejerce el apoyo social percibido, siendo éste un importante predictor del grado de ajuste general de los cuidadores (Heru y Ryan, 2006; Kinney y Parris Stephens, 1989; Pakenham, Chiu, Burnsall y Cannon, 2007; Rapp, Shumaker, Schmidt, Naughton y Anderson, 1998).

Los hallazgos hasta aquí expuestos son sólo algunos de los muchos que debemos al estudio de los aspectos positivos del cuidado. Sin embargo, esta consideración habitual de términos tales como los de satisfacción y beneficios del cuidado contrasta con la escasa investigación desarrollada en torno a la resiliencia, personalidad resistente y crecimiento en cuidadores, la cual se puede decir que se encuentra aún en una fase pionera. Pocos estudios han adoptado como tema central, y de modo directo, la capacidad del cuidador no profesional para resistir el estrés al que se somete y para encontrar en él una oportunidad para su propio desarrollo personal. A la escasez productiva al respecto hay que añadir, además, cierta dispersión y heterogeneidad en los datos aportados. 
Ante este panorama, el presente artículo tiene como finalidad precisamente ofrecer una revisión sistématica de los estudios sobre resiliencia, personalidad resistente (hardiness) y crecimiento efectuados en cuidadores no profesionales de mayores con demencia. La importancia de avanzar en esta dirección obedece a razones teóricas y prácticas. Estudiar la resiliencia, y conceptos asociados, en cuidadores permite, en primer lugar, situar la resiliencia dentro de un marco de estresores crónicos, ya que hasta la fecha la mayoría de las investigaciones publicadas la analizan en relación a potentes estresores puntuales. Además, a nivel clínico, conocer los factores que promueven la resiliencia en cuidadores permitirá la elaboración de programas de intervención preventivos, evitando la aparición de muchos de los problemas que con frecuencia se asocian al cuidado no profesional. Dichos programas, a su vez, podrán ir dirigidos a apuntalar y fomentar la capacidad del individuo no sólo para resistir la adversidad, sino para convertirla en una fuente de desafío y autorrealización personal, pudiendo constituir un complemento, cuando no una alternativa, a los tradicionales programas de tratamiento del malestar en cuidadores.

\section{Objetivos del presente trabajo}

A la luz de estas expectativas, y de forma específica, la revisión que se expone a continuación cubre un doble objetivo. El primero, sistematizar los datos encontrados hasta el momento, de modo que podamos conocer el porcentaje aproximado de cuidadores que se manifiestan resilientes (y resistentes) y, lo que es aún más importante, los factores que sirven para predecir estos resultados. No obstante, cabe advertir que la resiliencia es un constructo del que poco se conoce, a pesar de ser protagonista habitual de múltiples textos de psicología. Con el hardiness y el crecimiento personal sucede algo parecido. Por tanto, revisar los artículos donde estos términos son evaluados constituye un primer paso de suma relevancia para la construcción de modelos positivos útiles e integrados, siendo éste el segundo objetivo principal del presente artículo.

\section{Acercamiento conceptual a los términos resiliencia, personalidad resistente (hardiness) y crecimiento personal}

\section{El concepto de resiliencia}

A pesar de que el análisis de la resiliencia en cuidadores reviste un gran interés, toda investigación centrada en este constructo ha de enfrentarse a un buen número de dificultades. De todas ellas posiblemente la más relevante, y de la que derivan prácticamente todas las demás, es la relativa a la carencia de una definición de resiliencia debidamente consensuada y operativa (Becoña, 2006; Luthar, Cicchetti y Becker, 2000). Analicemos brevemente las tendencias teóricas más representativas en su estudio.

\section{Resiliencia como proceso o resultado}

Bonanno y Luthar, dos de los autores más relevantes en el campo, enuncian la resiliencia, respectivamente, como "una trayectoria estable de funcionamiento saludable a lo largo del tiempo, así como la capacidad para generar experiencias y emociones positivas" (Bonanno, 2004, p. 21), y como "un proceso dinámico que comprende la adaptación positiva dentro de un contexto significativamente adverso" (Luthar et al., 2000, p. 543).

Desde este planteamiento, la resiliencia aparece como el resultado de un exitoso proceso de adaptación (o como el mismo proceso) que incluye, además de la ausencia de sintomatología significati$\mathrm{va}$, aspectos relativos al adecuado funcionamiento de la persona. Además esa adaptación exitosa persiste a lo largo del tiempo, siendo este aspecto, precisamente, el que permite diferenciarla del concepto de recuperación (Bonanno, 2004). Es decir, diferencia de la resiliencia, la recuperación se refiere a un proceso de readaptación, el cual acontece necesariamente tras un periodo en el que el sujeto experimenta un estado físico o psicológico negativo.

Atendiendo a esta perspectiva, la resiliencia se circunscribiría a un momento temporal determinado, y se relacionaría con la exposición de la persona a una situación altamente estresante o de riesgo 
(Becoña, 2006; Masten, 2001; Luthar et al., 2000). El individuo, aunque ha de mostrar su competencia, no es en sí mismo portador de resiliencia, siendo ésta el fruto o la manifestación de la habilidad de la persona en interacción con el entorno. La resiliencia es, por lo tanto, un proceso dinámico, dependiente de las interacciones dadas entre múltiples variables, tanto internas como externas al propio individuo (Becoña, 2006; Olsson, Bond, Burns, VellaBrodorick y Sawyer, 2003).

\section{Resiliencia como capacidad}

Aunque las dos definiciones de resiliencia antes planteadas han obtenido una gran acogida, el acercamiento teórico probablemente más habitual, o al menos más difundido, sitúa la resiliencia en el plano intrapsíquico. De acuerdo con esta tendencia, la resiliencia es considerada como la capacidad o competencia del sujeto para hacer frente a situaciones desfavorables, siendo incluso concebida como un rasgo o característica de la personalidad del individuo (e.g. Block y Kremen, 1996). Dicha capacidad, lejos de ser excepcional, podemos encontrarla en un gran número de personas (Bonanno, 2004; Masten, 2001), aunque probablemente se manifieste de forma específica ante un tipo de eventos y no ante otros (Masten, 2001).

La concepción de la resiliencia como capacidad ha posibilitado el desarrollo de escalas específicas para su medida, las cuales dependen a menudo de la valoración de una serie de aptitudes o capacidades de menor orden del sujeto. De hecho, la escala de resiliencia más comunmente empleada en la actualidad, la de Connor-Davidson (Connor-Davidson Resilience Scale. CD-RISC. Connor y Davidson, 2003), evalúa 5 factores considerados componentes esenciales de la resiliencia (competencia personal, confianza en la propia intuición y tolerancia a la adversidad, aceptación positiva del cambio, control, y espiritualidad). Puntuaciones elevadas en medidas de este tipo no garantizan, sin embargo, que la persona no desarrolle sintomatología psicológica ante la presencia de un estresor severo. De acuerdo con esta tendencia, altos niveles de resiliencia sólo permiten predecir en el sujeto una menor probabilidad de presentar cuadros de naturaleza psicopatológica.

En suma, desde este punto de vista podemos definir la resiliencia como la capacidad del sujeto para afrontar una situación vital estresante sin que su salud física y psicológica se vea gravemente comprometida, ni su funcionamiento habitual alterado. No obstante, esta consideración de la resiliencia como característica inherente a la persona facilita su solapamiento con el concepto de personalidad resistente o hardiness (dureza), hasta el punto de considerarse a veces como términos intercambiables.

\section{La personalidad resistente o hardiness}

Kobasa y Maddi son los primeros en referirse al hardiness, en 1972, para explicar cómo determinadas personas poseen la capacidad de resistir el estrés con mayor éxito que otras. Según Kobasa (1979) hay diferencias estructurales de personalidad que conllevan a que en determinadas situaciones unos individuos enfermen y otros no, y tales diferencias constituyen la personalidad resistente.

Desde su nacimiento la noción de personalidad resistente se ha enmarcado dentro del movimiento existencialista (Peñacoba y Moreno, 1998), entendiéndose como una manera de concebir la vida que implica, según Maddi (2008), la asunción del riesgo a favor del crecimiento personal. Esta manera de afrontar el destino requiere que se reúnan tres características o dimensiones del sujeto, a saber: compromiso, desafio y control. El factor compromiso hace referencia a la habilidad de algunas personas para implicarse hondamente en las actividades de su vida cotidiana; desafio representa la capacidad para experimentar el cambio como una oportunidad para el crecimiento; y, por último, control, la creencia de que las circunstancias de la vida dependen de las propias acciones. Esto implica que la persona resistente, ante un estresor, realizará una serie de reajustes vitales que favorecerá su adaptación. Para ello habrá de mantener presentes en todo momento su sistema de metas y valores; considerar sus capacidades personales; adoptar con vigor las decisiones oportunas; evaluar las repercusiones en términos de sus propias conductas (estableciendo un locus de 
control interno); y dotar a la nueva situación de un significado, es decir, darle un sentido dentro de su plan general de vida (Kobasa, 1979).

\section{Diferencias entre la resiliencia y la personalidad resistente}

En definitiva, la personalidad resistente, de modo similar a la resiliencia, implica una capacidad para hacer frente de forma adaptativa a las circustancias adversas, con evidentes repercusiones en el estado emocional, y también físico, del individuo. No obstante, la personalidad resistente parece hacer alusión a un macrofactor de personalidad, compuesto por las tres dimensiones descritas (compromiso, desafío y control) (Kobasa, 1979), dimensiones que puede comprender la resiliencia pero no de modo exclusivo. De acuerdo con esto, la principal diferencia perceptible entre la resiliencia y la personalidad resistente es que la primera engloba algo más que la segunda.

En decir, el hardiness, que emerge como un patron de actitudes y comportamientos, mejora la resiliencia del sujeto ante situaciones potencialmente estresantes, constituyendo tan sólo una de las posibles vías de desarrollo de resiliencia (Bonanno, 2004; Maddi, 2005). De esta forma, aquellas personas que alcancen elevados niveles de hardiness tendrán una mayor probabilidad de ser resilientes, fenómeno que no se producirá necesariamente a la inversa.

\section{El crecimiento ante la adversidad}

A menudo los individuos, además de resistir con insospechada entereza las situaciones más desfavorables, encuentran en la adversidad una oportunidad para su crecimiento personal. Dicho crecimiento se refiere al "cambio psicológico positivo experimentado como resultado de la lucha ante circunstancias de la vida altamente desafiantes" (Tedeschi y Calhoun, 2004, p.1). Es, por tanto, fruto del intento del sujeto por afrontar los eventos a los que se expone, no siendo producido por el acontecimiento traumático en sí mismo (Tedeschi y Kilmer, 2005).
Además, el crecimiento no es incompatible con el padecimiento de malestar o sufrimiento, siempre y cuando el individuo obtenga beneficios personales de la experiencia adversa vivida (Tedeschi y Kilmer, 2005).

Calhoun y Tedeschi (2000) distinguen tres tipos de cambios que puede experimentar el individuo, los cuales actúan como indicadores de crecimiento tras el trauma: (a) cambios en relación a uno mismo (aumento de la confianza en sí mismo y de la percepción de la propia fortaleza), (b) cambios en la espiritualidad y filosofía de la vida (referidos a la escala de valores y prioridades vitales), y (c) cambios a nivel interpersonal (consolidación de vínculos sociales, expresiones afectivas, etc.).

Aunque el crecimiento postraumático requiere, como su propio nombre indica, la presencia de un acontecimiento traumático, es un término extensible al contexto de los estresores crónicos, tal como la situación de cuidado, donde el estrés prolongado también ofrece la oportunidad al sujeto de experimentar cambios positivos a nivel personal y social (e.g. Phelps et al., 2009, encuentran distintos niveles de crecimiento en padres que asisten a sus hijos autistas). Por ello, aludiremos a partir de ahora a la noción de crecimiento personal, evitando las reminiscencias al concepto de trauma.

\section{Características específicas del crecimiento personal}

Si bien los conceptos de resiliencia y hardiness se intercambian con frecuencia, la consideración de la noción de crecimiento añade aun una mayor confusión terminólogica. Para su correcta delimitación, es necesario tener en cuenta que el crecimiento siempre exige que se produzca un cambio positivo en el individuo, mientras que la resiliencia alude al funcionamiento estable del sujeto ante un estresor sin necesidad de que opere un proceso de cambio. Cierto es, por otro lado, que la definición del hardiness que aporta Maddi (2008) (asunción de riesgo a favor del crecimiento personal) ya engloba la idea de crecimiento, lo cual no ayuda a dilucidar las diferencias entre estos términos. No obstante, podemos observar que el crecimiento personal 
aparece aquí como una probable consecuencia del hardiness, de modo que la consideración de las circustancias en términos de compromiso, desafío y control favorecerá la experiencia de crecimiento personal.

A modo de resumen, se podrían establecer las siguientes relaciones: la personalidad resistente (integrada por los componentes compromiso, desafío y control) aparece como una posible vía de desarrollo de la capacidad de resiliencia (dependiente además de otros factores), cuyo desarrollo irá dirigido a promover el crecimiento personal (concebido ahora como resultado). Aclarados estos conceptos, pasemos a analizar cómo han sido aplicados a la población de cuidadores no profesionales de mayores con demencia.

\section{Revisión bibliográfica de estudios que analizan la resiliencia, el hardiness y el crecimiento en cuidadores no profesionales de mayores con demencia}

A fin de efectuar un análisis sistemático de los estudios dirigidos a valorar la resiliencia, la personalidad resistente y el crecimiento en cuidadores no profesionales se llevó a cabo una búsqueda bibliográfica de los artículos publicados al respecto. Para esta exploración bibliográfica se hizo uso de las bases de datos PsycINFO y MEDLINE. La selección de artículos no se vio sometida a ningún tipo de restricción temporal. Asimismo éstos fueron considerados con independencia del país y la organización responsable de su publicación. Los identificadores empleados en la búsqueda fueron las palabras resilience, resiliency, hardiness, growth, caregiver, caregivers, care y caregiving, disponiéndose todas las combinaciones posibles entre ellas. Una vez presentada la lista de artículos adecuados a las condiciones de búsqueda, se seleccionaron, de modo específico, los que incluían algún estudio empírico prestando epecial atención al empleo de análisis de regresión con cuidadores no profesionales de mayores con demencia.

Como resultado del procedimiento descrito se identificaron nueve artículos de interés, siete de ellos realizados en Estados Unidos (Clark, L. y Hartman, 1996; Clark P., 2002; DiBartolo y Soeken,
2003; Gaugler, Kane y Newcomer, 2007; Ott, Sanders y Kelber, 2007; Wilks y Croom, 2008; Wilks y Vonk, 2008), uno en Alemania (Leipold, Schacke y Zank, 2008) y otro en Canadá (Milne, Sacco, Cetinski, Browne y Roberts, 1994). La información contenida en dichos artículos aparece resumida en la Tabla 1, en la cual se presentan ordenados alfabéticamente y considerando para cada uno: (1) Identificación del trabajo (autores y año de publicación); (2) Número de participantes y características (edad, género y parentesco con la persona cuidada); (3) Concepto y medidas de evaluación de la resiliencia, la personalidad resistente y el crecimiento personal; (4) Variables con las que se relacionan y (5) Resultados encontrados.

Cabe reseñar que todos los estudios son de publicación reciente: el más remoto data de 1994, habiendo sido todos publicados en torno a los últimos 15 años. Ello sugiere que el interés por los aspectos positivos del cuidado, concretamente el interés científico por la resiliencia, el hardiness y crecimiento en cuidadores no profesionales, es relativamente reciente, si bien no hay que olvidar que la publicación de este tipo de investigaciones sigue siendo escasa aún en la actualidad.

\section{Caracterización de los estudios}

En primer lugar, observamos que todos los estudios seleccionados emplean muestras de conveniencia, a excepción del trabajo de Gaugler et al. (2007), en el que se utiliza una muestra aleatoria entre los participantes en un programa de seguimiento de caso para cuidadores de personas con Alzheimer u otras demencias. La recogida de la información se efectuó fundamentalmente o bien a través de entrevistas directas (e.g. Clark P., 2002) o bien por correo ordinario (e.g. DiBartolo y Soeken, 2003).

Aunque el número de participantes es desigual, en general, las muestras recogidas tienen un tamaño medio, incluso pequeño: en cuatro estudios se sitúan por debajo de los 100, mientras que en uno de ellos (Gaugler et al., 2007) se parte de una muestra inicial de casi 2.000 sujetos.

Las muestras estaban compuestas mayoritariamente por cuidadores primarios, los cuales podían o 


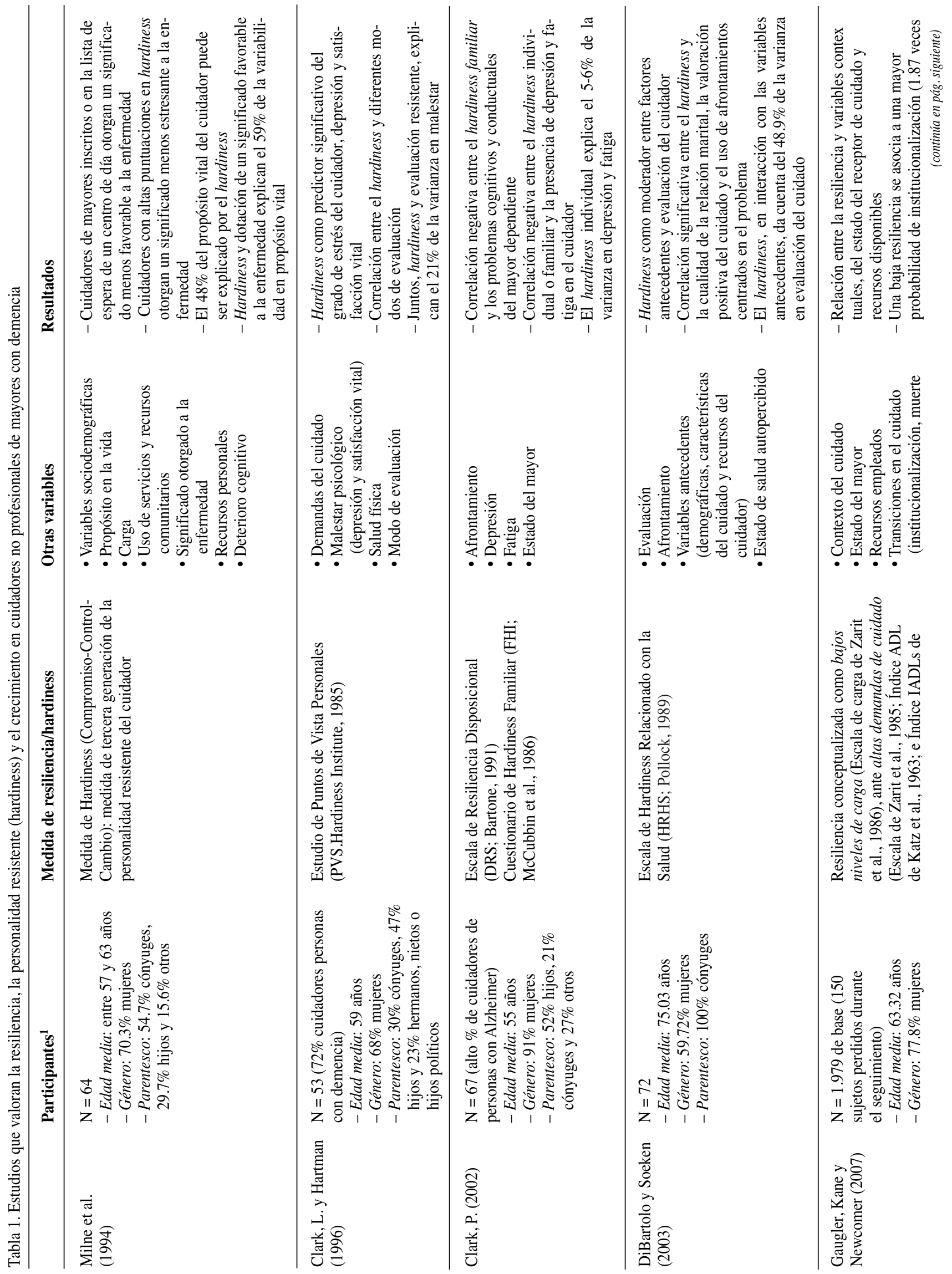




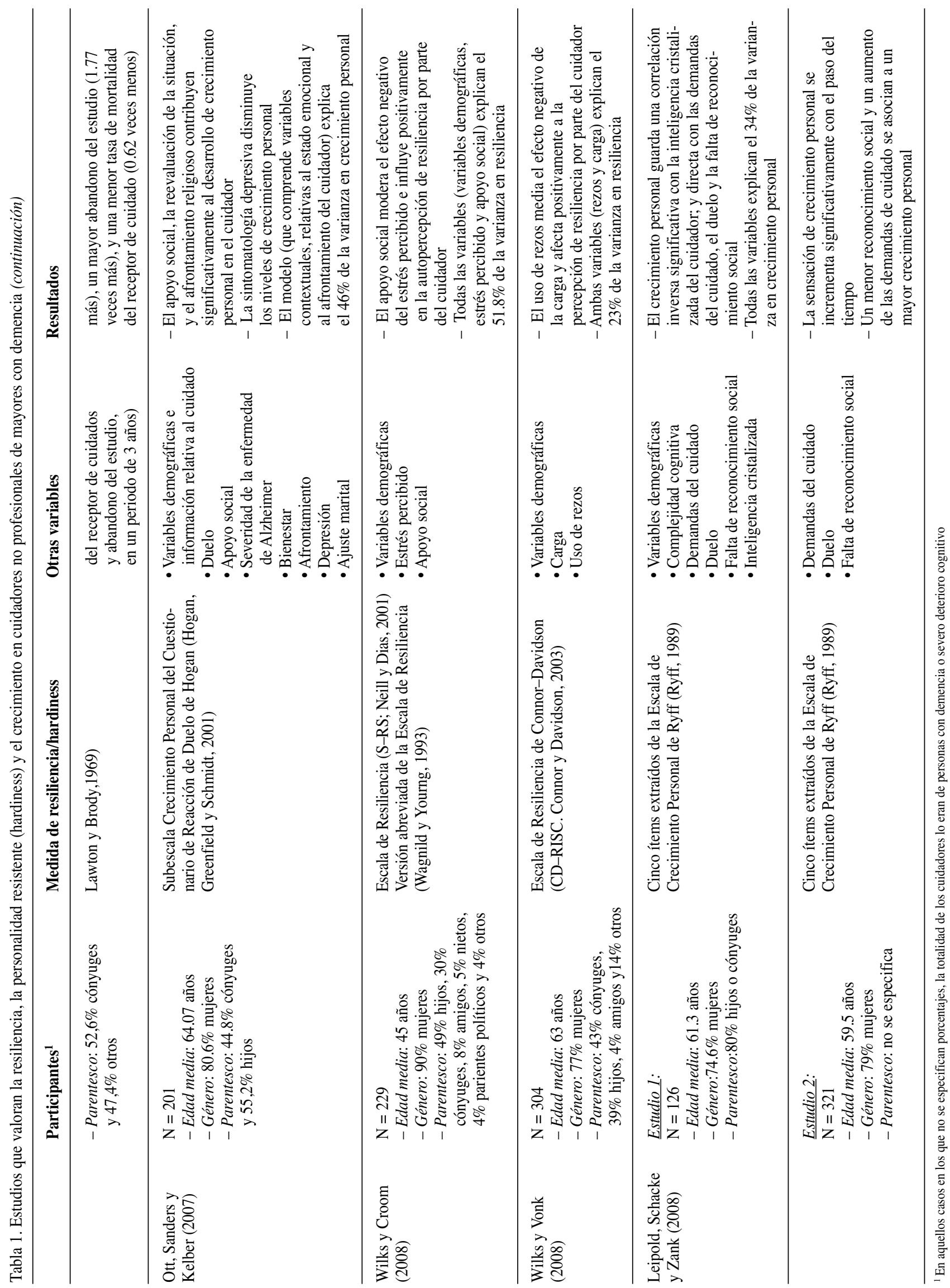


no convivir con el receptor de cuidado. Estos cuidadores fueron, en la casi totalidad de los casos, cónyuges o hijos de la persona cuidada. Se observa que el número de mujeres cuidadoras es siempre superior al de varones, y que el rango de edad de los participantes se sitúa entre los 32 y los 84 años (aunque con una mayor concentración en torno a los 50-60 años), lo cual se corresponde con el perfil general de los cuidadores, tanto en nuestro país (e.g. IMSERSO, 2005), como en otros contextos, por ejemplo Estados Unidos (e.g. Baker y Robertson, 2008).

En cuanto a los receptores del cuidado, éstos fueron, dados los propios criterios de inclusión, personas mayores ${ }^{1}$ que padecían algún tipo de deterioro cognitivo. Sus edades estaban comprendidas fundamentalmente entre los 75 y los 97 años, y la amplia mayoría eran mujeres. Este último dato puede ser explicado si tenemos en cuenta que cuanto mayor sea la edad, más probable es el desarrollo de una demencia que dificulte la autonomía de la persona, y que la esperanza de vida de las mujeres supera a la de los varones (Encuesta de Discapacidad, Autonomía personal y situaciones de Dependencia. EDAD, 2008). Es decir, presumiblemente serán las mujeres las que lleguen a una edad más avanzada y, por ello, desarrollen un mayor número de alteraciones cognitivas o alcancen estadios más avanzados de deterioro.

Por otro lado, se observa que los estudios utilizan preferentemente diseños transversales con medida única de las variables evaluadas. Las únicas excepciones las constituyen los trabajos de Leipold et al. (2008), quienes efectúan un seguimiento de los participantes a lo largo de 27 meses; y de Gaugler et al. (2007), en el que la medida de las variables de transición del cuidado (e.g. institucionalización o muerte del receptor de cuidados), por su propia naturaleza, se realiza cada 6 meses durante un periodo de 3 años, si bien el resto de variables se toman en una sola evaluación inicial. Además, Milne et al. (1994)

\footnotetext{
${ }^{1}$ Aunque se asume que los receptores de cuidado fueron personas mayores, existen diferencias entre estudios en cuanto al establecimiento del límite de edad. En aquellas publicaciones en las que no se incluyen especificaciones al respecto se indica como requisito la presencia de Alzheimer, otras demencias o deterioro cognitivo, por lo que se deduce que la edad media de los receptores de cuidado será avanzada.
}

hace uso de un diseño comparativo de grupo, aunque sin incluir datos de seguimiento.

En todos los casos se trata de estudios correlacionales que utilizan algún procedimiento de análisis de regresión para la consideración de los resultados, tal y como se establecía en los criterios de selección de estudios. Dos de los trabajos, de los más recientes, analizan además las razones de ventaja u odds ratio (Gaugler et al., 2007; Wilks y Croom, 2008).

La conceptualización y las medidas empleadas para evaluar la resiliencia, la personalidad resistente o la experiencia de crecimiento de los participantes, difieren sustancialmente entre estudios. Esto es, no se dispone de un instrumento consensuado para valorar dichos constructos y, lo que resulta aún más llamativo, ni siquiera se dispone de un modelo teórico integrado. Así, mientras que algunos autores hacen uso de escalas específicas de resiliencia (e.g. la escala S-RS de Neill y Dias, 2001 o la CD-RISC de Connor y Davidson, 2003) y hardiness (e.g. la escala PVS del Hardiness Institute, 1985), otros prefieren definir la resiliencia como el resultado de las puntuaciones del sujeto en una o más variables relacionadas. Así, Gaugler et al. (2007) entienden la resiliencia como la conjunción de bajos niveles de carga (evaluada a través de la Escala de carga de Zarit et al.,1986), ante altas demandas de cuidado (valoradas en virtud del deterioro y problemas presentes en los receptores de cuidado). Por su parte, las dos investigaciones que consideran el crecimiento personal de los participantes (Leipold et al., 2007; Ott et al., 2007) lo hacen a través de escalas diseñadas a este propósito (la Escala de crecimiento personal de Ryff, 1989; y la subescala de crecimiento personal del Cuestionario de reacción de duelo de Hogan, 2001; respectivamente).

Para terminar, las variables incluidas en cada estudio se basan en modelos teóricos que proporcionan un marco conceptual de referencia a la hora de establecer relaciones entre ellas. Grosso modo, estos modelos se enmarcarían dentro de los transaccionales de estrés (derivados del modelo de Lazarus y Folkman, 1984), si bien existen diferencias entre estudios. En cualquier caso, y de forma general, todos integran los conceptos de resiliencia, personalidad resistente y crecimiento en los modelos existentes, estableciendo relaciones y posibles solapa- 
mientos entre éstos y las variables incluidas en dichos modelos: variables contextuales, estresores, evaluación de los estresores, mediadores del estrés (e.g. afrontamiento o apoyo social) y resultados en el estado emocional y físico del cuidador.

\section{Resultados de los estudios y conclusiones}

\section{¿Existen cuidadores resilientes? ¿Y resistentes?}

De manera general, los estudios analizados constatan la presencia de resiliencia en cuidadores de personas con demencia en el entorno familiar. Así, la resiliencia mostrada por los participantes alcanza niveles moderados-altos en los estudios de Wilks y Croom (2008) y de Wilks y Vonk (2008), estando presente en el $45.3 \%$ de los cuidadores evaluados por Gaugler et al. (2007).

En cuanto a la personalidad resistente, los datos son aún más concluyentes, ya que su distribución en las muestras de cuidadores es similar a la de la población general (Clark, L. y Hartman, 1996), logrando los participantes niveles entre moderados y elevados de hardiness (DiBartolo y Soeken, 2003; Milne et al., 1994).

Es de resaltar, sin embargo, que la presencia de resiliencia y hardiness no es incompatible con la presencia de malestar y problemas emocionales, puesto que en estos mismos estudios se han encontrado niveles importantes de carga percibida (Milne et al., 1994; Wilks y Vonk, 2008), estrés (Wilks y Croom, 2008), depresión (Clark, L. y Hartman, 1996; Clark P., 2002) y fatiga (Clark P., 2002). En consecuencia, aunque la resiliencia y el hardiness tienden a relacionarse de modo inverso con los efectos negativos del cuidado, altas puntuaciones en cualquiera de las dos variables no permiten predecir de modo absoluto la ausencia de carga en el cuidador u otro tipo de sintomatología.

\section{¿Qué variables se asocian a la resiliencia en cuidadores?}

Las variables que contribuyen al desarrollo de elevados niveles de resiliencia en el cuidador son múltiples y de naturaleza variada. Agrupando los principales factores que interactúan con la resiliencia del sujeto, Gaugler et al. (2007) dibujan tres costelaciones de variables, a saber: (a) el contexto del cuidado (referido a la edad, raza, relaciones familiares, género y estatus socioeconómico del cuidador), (b) el estado del mayor (daño cognitivo, necesidades de cuidado y horas de cuidado), y (c) los recursos familiares, individuales y comunitarios (apoyo secundario, servicios comunitarios, funciones del cuidador y recursos intrapsíquicos).

En cuanto a las dos primeras categorías, y en consonancia con sus hipótesis iniciales, Gaugler et al. (2007) han encontrado que el contexto del cuidado y el estado del mayor tienen efectos en la evaluación por parte del cuidador del estrés experimentado, así como en la manifestación de las demandas de la situación. Cuidadores que hayan desempeñado esa tarea durante un tiempo prolongado, y atiendan a mayores del sexo femenino, con un menor nivel de deterioro cognitivo o una menor necesidad de asistencia, indicarán una mayor resiliencia. Además, también favorecerá la resiliencia una serie de características sociodemográficas relativas al propio cuidador, tales como el hecho de ser latino o afroamericano. Esto lo explican los autores en función de las creencias culturales y la socialización del sujeto, así como en función de la adaptación longitudinal a su rol de cuidador.

Entre aquellas variables que afectan negativamente a la resiliencia de la persona, Gaugler et al. (2007) incluyen, en contra de lo esperado, un nivel elevado de ingresos económicos y un alto nivel educativo, además de la raza caucásica del cuidador. El controvertido papel que ejerce el nivel socioeconómico y educativo del cuidador sobre su resiliencia puede explicarse debido a que personas con elevados ingresos probablemente opten en mayor medida por convivir con el receptor de cuidado (al poder hacerse cargo de los gastos que esto ocasiona) y se vean, por ello, expuestas a un alto grado de demandas.

Por otro lado, diversos estudios coinciden en destacar la importancia del vínculo existente entre el apoyo social recibido por el cuidador (ya sea familiar o por parte de sus amigos) y su resiliencia, ejerciendo, el apoyo social, la función de amortiguador 
del efecto del estrés sobre el estado emocional de la persona, y siendo este efecto mayor en el caso del apoyo social familiar (Wilks y Croom, 2008). Wilks y Croom (2008) proponen distintas vías por las cuales puede operar esta relación, no excluyentes entre sí. En primer lugar, el estrés podría generar una percepción distorsionada acerca de los recursos sociales disponibles; o, en segundo lugar, influir en la habilidad del cuidador para hacer uso de esos recursos. Por último, precisamente podría ser la percepción de poseer una sólida fuente de apoyo social lo que contribuyese a generar una menor sensación de estrés en el cuidador.

No obstante, y a pesar de que las características relativas al contexto de cuidado y al apoyo social ejercen una influencia innegable sobre la resiliencia del cuidador, parece que son sus propios recursos intrapsíquicos (autoeficacia, autoestima, habilidad, etc.) los que con mayor fuerza se asocian, ya sea como predictores o consecuencias, a dicha variable. En esta línea, el tipo de evaluación y el estilo de afrontamiento empleado por el sujeto se han constituido como factores de importancia capital para el desarrollo de altos niveles de resiliencia. Cabe destacar, a pesar de no estar incluida en la revisión ${ }^{2}$, la investigación emprendida por Garity (1997), quien concluye que los cuidadores resilientes utilizan predominantemente afrontamientos centrados en el problema y tienden a no hacer uso del escape o la evitación. Además el uso de plegarias o rezos puede ser considerado como un estilo particular de afrontamiento, que sirve asimismo para favorecer la resiliencia del individuo (Wilks y Vonk, 2008).

De igual forma, se cree que la resiliencia del cuidador afecta al modo en que éste evalúa el estrés que experimenta, de manera que los cuidadores resilientes efectúan una valoración más benigna de los estresores asociados a la asistencia, lo cual, a su vez, tendrá importantes repercusiones en su salud mental (Gaugler et al., 2007). Braithwaite (2000) ha denominado estilo de evaluación resistente a aquel en el que predomina la eficacia y fortaleza, resultado de la conjunción de una serie de recursos personales.

${ }^{2}$ El estudio de Garity (1997) no ha sido incluido en la revisión bibliográfica porque el autor emplea exclusivamente estrategias de análisis de tipo correlacional, menos potentes que las estrategias multivariadas utilizadas por el resto de artículos considerados en la presente revisión.

\section{¿Qué factores correlacionan con el hardiness?}

Las variables que han sido destacadas a la hora de predecir la construcción de una personalidad resistente no distan mucho de las variables que tradicionalmente se han asociado al desarrollo de altos niveles de resiliencia. No en balde se señalaba en apartados anteriores que el concepto de resiliencia guarda un estrecho vínculo con el de hardiness. Así, los artículos recogidos en torno a la personalidad resistente subrayan su asociación con variables relativas al contexto del cuidado, al estado del mayor, al apoyo social, $\mathrm{y}$, fundamentalmente, a los recursos intrapsíquicos del cuidador.

En relación al estado del receptor de cuidado, los hallazgos de Clark, P. (2002) revelan que los problemas de conducta y memoria manifiestos por el enfermo guardan una correlación inversa significativa con el hardiness familiar, siendo además éstos los que con mayor peso contribuyen a predecir la percepción de carga del cuidador. Según la autora, es posible que las familias resistentes empleen técnicas más efectivas para la solución de problemas relacionados con la asistencia, lo cual podría repercutir positivamente en el estado del mayor dependiente. Alternativamente, las familias incapaces de lidiar con los problemas mostrados por el receptor de cuidado podrían ver disminuido su nivel de resistencia, como consecuencia.

Además los datos de los que disponemos muestran que la cualidad de la relación marital entre el cuidador y el receptor de cuidado correlaciona significativamente con el hardiness, de modo que los cónyuges que establezcan un vínculo más estrecho con el mayor serán, asimismo, los más resistentes (DiBartolo y Soeken, 2003). Milne et al. (1994) encuentran, también, que cuidadores que conviven con el receptor de cuidado tienen un menor índice de hardiness, lo cual concuerda con los resultados hallados por Gaugler et al. (2007) en relación a la resiliencia.

A pesar de la importancia para el hardiness de algunas características objetivas de la situación de cuidado, y de la relación establecida entre el cuidador y el mayor dependiente, son nuevamente el modo de evaluación de la situación de asistencia $\mathrm{y}$ el estilo de afrontamiento empleado por el 
cuidador los dos aspectos en los que más insisten los investigadores interesados por este campo (advirtamos que Clark, 2002, ya recurre al estilo de afrontamiento para explicar la relación entre el hardiness y los problemas presentes en el mayor). De hecho, a menudo el propio concepto de hardiness encierra modos de evaluación y afrontamiento particulares. No obstante, el papel otorgado a ambos factores será diferente según el modelo teórico que se adopte.

En referencia al tipo de evaluación, Milne et al. (1994) obsevan que el hardiness es el factor que con mayor fuerza predice la variable propósito en la vida. Es decir, cuidadores resistentes, y cuidadores que otorguen un significado menos estresante a la situación de asistencia, tenderán a experimentar en mayor grado la sensación de que su vida tiene un sentido. En otro orden, cuidadores que utilizan o se hallan en la lista de espera de un centro de día otorgan un significado menos favorable a la enfermedad en comparación con cuidadores que rechazan dicho recurso o han institucionalizado al mayor.

Clark y Hartman (1996), aplicando el Estudio de Puntos de Vista Personales (PVS. Hardiness Institute, 1985) (y partiendo de la consideración de que un afrontamiento resistente se caracterizará por la percepción de que uno mismo es responsable y puede ejercer su influencia sobre la situación de cuidado -evaluaciones de control-, la consideración de que el cuidado constituirá una posibilidad para su crecimiento personal -evaluaciones de cambio o desafio-, y la percepción de que esta experiencia goza de un significado valioso para el individuo -evaluaciones de compromiso-), encuentran que, si bien el tipo de evaluación realizado por el sujeto media su grado de resistencia, la conjunción de ambas variables (hardiness y estilo de evaluación resistente) da cuenta de las diferencias en salud mental y, en menor medida, de distintos aspectos relacionados con la salud física de los cuidadores. Es decir, aquellos cuidadores que evalúen positivamente su situación tenderán a desarrollar menores niveles de depresión y verán menos comprometida su satisfacción vital, además de poseer un menor número de síntomas físicos. En concreto, los autores hallan que el hardiness y el estilo de evaluación del cuidador actúan como potentes predictores de su estado psicólogico, una vez han sido controladas las demandas del cuidado. No obstante, y a pesar del solapamiento parcial entre el rasgo de personalidad resistente y los estilos de evaluación resistentes, lo autores advierten que éstos no pueden considerarse sinónimos. Es más, la personalidad resistente no ejerce un impacto determinante sobre el modo de evaluación del cuidador, refiriéndose ésta a un rasgo general del sujeto que no tiene porqué traducirse en una situación tan concreta como la del cuidado. Además la personalidad resistente parece que afectaría al estado psicológico del sujeto a través de mecanismos adicionales, tales como las estrategias de afrontamiento empleadas por éste.

Posteriormente, DiBartolo y Soeken (2003) estudiarán nuevamente la relación dada entre el modo de evaluación y la resistencia del sujeto, aunque esta vez la personalidad resistente sí se considerará como una variable moderadora entre los antecedentes del cuidador (variables demográficas, características del cuidado y recursos del cuidador) y la forma en que evalúe la situación de cuidado. Es decir, las personas más resistentes tenderán a evaluar más positivamente esta experiencia, lo cual quizás explique una percepción más optimista de su relación conyugal. Además el hardiness también determinará el estilo de afrontamiento de la persona, haciendo uso los individuos más resistentes de estrategias de afrontamiento focalizadas en el problema.

Coincidiendo con sus predecesores, Clark, (2000) sostiene que quienes obtengan puntuaciones más elevadas en hardiness, tanto a nivel individual, como familiar, emplearán estrategias de afrontamiento más efectivas, destacando el uso de un estilo de afrontamiento transformacional, y recurrirán en mayor medida a la búsqueda de ayuda.

\section{¿Se asocian la resiliencia y/o la personalidad resistente a un adecuado estado de salud mental?}

Como se ha señalado en párrafos anteriores, la investigación corrobora que tanto la resiliencia como la resistencia del cuidador guardan una 
estrecha correlación negativa con el desarrollo de sintomatología psicopatológica y permiten predecir distintos aspectos relativos a las transiciones del cuidado. Es más, Gaugler et al. (2007) exigen, como parte integrativa del concepto de resiliencia (coincidiendo con las posturas que entienden la resiliencia como resultado) que se den bajos niveles de carga en el cuidador.

Además, el estudio de Gaugler et al. (2007) establece que la clasificación de los cuidadores en alta y baja resiliencia permite predecir el modo en que éstos se adaptarán a las distintas fases de su situación. Así, en este trabajo, los cuidadores menos resilientes tendieron en mayor medida a renunciar a su rol, a institucionalizar al enfermo y a abandonar el estudio. Inesperadamente, los cuidadores resilientes asistieron con mayor frecuencia al fallecimiento del mayor. Una explicación tentativa a este último hallazgo sugiere que quizás aquellos cuidadores que asisten a personas con un alto grado de deterioro y, por tanto, con una muerte más próxima, experimenten cierta sensación de alivio debido a la anticipación del fin de su labor. De este modo, cuidadores de ancianos que se encuentren en un estadio más avanzado de demencia serán más resilientes, lo que vendría a apoyar la hipótesis de adaptación al cuidado frente a la de desgaste progresivo (wear and tear) (e.g. Townsend, Noelker, Deimling y Bass, 1989).

Por otro lado, fruto de la exploración de las relaciones entre la personalidad resistente y el desarrollo de sintomatología, los distintos estudios constatan que elevados niveles de hardiness en cui-dadores se asocian a una menor sensación subjetiva de carga, estrés y fatiga (Clark, 2002; Milne et al., 1994; Wilks y Croom, 2008; Wilks y Vonk, 2008), a menores niveles de depresión (Clark y Hartman, 1996; Clark, 2002) y a altos niveles de satisfacción (Clark y Hartman, 1996). Sin embargo, una vez más, los autores no se ponen de acuerdo a la hora de explicar el modo en que interactúan estas variables, si bien es probable que la personalidad resistente y el estado de salud mental del cuidador ejerzan una influencia recíproca entre sí, viéndose los resultados obtenidos en cada variable alimentados por los resultados obtenidos en la otra.
Un paso más allá:; es posible el crecimiento personal a través del cuidado?

Además de mostrar niveles moderados (o elevados) de resiliencia y hardiness, los familiares de mayores dependientes también describen experiencias de crecimiento personal derivadas de la asunción de su rol de cuidador. Estas experiencias implican una serie de cambios psicológicos en el cuidador, entre los que se incluyen el aumento de sentimientos de compasión, tolerancia o esperanza (Ott et al., 2007), e importantes cambios perceptivos en relación a sí mismos y su situación vital (Leipold et al., 2008).

Adicionalmente, el crecimiento personal parece incrementarse con el paso del tiempo. Así, en una primera toma de información Leipold, et al. (2008) encuentran una puntuación media de 2.59 en los cinco ítems de crecimiento que seleccionan de la escala de Ryff (1989), mientras que, transcurridos 27 meses, la puntuación media obtenida por los participantes de este estudio asciende a 2.84 .

\section{¿A qué se asocia la experiencia de crecimiento?}

La coexistencia de experiencias de crecimiento personal con distintos problemas psicológicos resulta aún menos llamativa que en el caso de la resiliencia o el hardiness, ya que en la propia conceptualización de esta variable se exige a menudo que se produzca un sufrimiento emocional, a través del cual el individuo extraiga beneficios para su propio desarrollo personal (Tedeschi y Calhoun, 2004). Es más, los resultados obtenidos por Leipold et al. (2008) apuntan a que mayores niveles de duelo en cuidadores predecirán una mayor sensación de crecimiento, crecimiento que aumentará además a medida que se incrementan las demandas de cuidado.

En relación a la depresión, sin embargo, los resultados hallados apuntan en la dirección opuesta, considerando Ott et al. (2007) que elevados niveles de sintomatología depresiva disminuyen los niveles de crecimiento personal. La discrepancia entre autores en este punto, puede solventarse a la luz del siguiente razonamiento: El duelo se refiere a la pérdida paulatina del familiar, cuyas características más 
relevantes de personalidad se van desdibujando a medida que progresa la enfermedad. Ante esta pérdida anticipada, el cuidador puede desarrollar nuevas cogniciones dirigidas a aliviar su malestar, las cuales pueden traducirse en experiencias de crecimiento personal. El desarrollo de un cuadro depresivo, sin embargo, podría ser el resultado del fracaso del tal estrategia de defensa.

En cualquier caso, a diferencia de como sucede con la personalidad resistente y la resiliencia, Leipold et al. (2008) vinculan el crecimiento a situaciones altamente demandantes, en las que los cuidadores se hayan sometidos a fuertes estresores. Adicionalmente, la falta de reconocimiento social percibido por el cuidador facilitará la vivencia de experiencias de crecimiento personal, derivadas quizás, tal y como señalan los propios autores, del intento del sujeto por resolver la disonancia producida entre la ausencia de reconocimiento externo y la importancia que el mismo concede a su rol. El apoyo social que recibe el cuidador, sin embargo, sí parece contribuir significativamente a su crecimiento personal (Ott et al., 2007).

Otras variables que favorecen el crecimiento en cuidadores son la reevaluación o replanteamiento del problema (coping by reframing) y la religiosidad (Ott et al., 2007), siendo éstas estrategias de afrontamiento eficaces para hacer frente a la situación de asistencia y dotarla, así, de transcendencia o significado. Por último, el crecimiento ha sido relacionado negativamente con la inteligencia cristalizada. Leipold et al. (2008) encuentra en el mayor excepcismo de las personas más inteligentes una plausible explicación a este fenómeno.

\section{Limitaciones encontradas en los estudios}

Aunque los datos aportados por los trabajos analizados permiten extraer conclusiones relevantes, en todos estos estudios se observan algunas limitaciones, reconocidas en muchos casos por los propios autores. Pinquart y Sörensen (2003a) ya señalaron, al efectuar su conocido meta-análisis sobre efectos negativos del cuidado, que a menudo las muestras analizadas no tienen el tamaño requerido o no son lo suficientemente representativas como para poder extraer conclusiones generalizables. A esto se suma la escasez de trabajos con cuidadores que incluyan diseños metodológicos longitudinales, teniendo la mayoría un corte transversal (Goode, Haley, Roth y Ford, 1998; Liming Alspaugh, Zarit y Greene, 1999). Con la resiliencia, la personalidad resistente y el crecimiento, no sucede algo diferente. Exceptuando la investigaciones de Gaugler et al. (2007) y de Leipold, et al. (2008), todas las aquí recogidas parten de muestras de tamaño medio, o incluso pequeño, y carecen de medidas para observar la evolución de los sujetos. Otras limitaciones comunes entre estudios se refieren a la utilización de muestras de conveniencia, la ausencia de datos que permitan establecer comparaciones interculturales e intersexo (siete de los nueve trabajos están realizados en Estados Unidos y con una amplia mayoría de participantes del sexo femenino), la falta de grupos control, o la frecuente confusión entre tipos de factores (e.g. de protección, de riesgo, mediadores o moduladores). Además en la mayoría de los estudios se echa en falta la evaluación de algunas variables (e.g. satisfacción con el cuidado) cuyo efecto podría contribuir considerablemente a explicar los resultados obtenidos.

No obstante, la mayor limitación que se observa, fundamentalmente en relación al estudio de la resiliencia, se refiere a la heterogeneidad en cuanto al planteamiento teórico de partida. A modo de ejemplo, de todos los trabajos analizados es el de Gaugler et al. (2007) el que goza de mayor relevancia a día de hoy, ya que plantea un modelo específico de resiliencia en cuidadores (que contempla diversas variables pertenecientes a distintos dominios), adaptado del modelo multidimensional propuesto por Bergeman y Wallace (1999). Sin embargo, la aplicabilidad de este modelo queda limitada al contemplar la sorprendente definición de resiliencia que proponen los autores, la cual difiere sustancialmente de las que parten el resto de los autores. En concreto, Gaugler et al. (2007) entienden la resiliencia en función de la presencia de varios niveles de carga en relación a las demandas de cuidado (bajos niveles de carga ante altas demandas de cuidado), y es preciso tener en cuenta que la resiliencia nunca debe entenderse como el opuesto a la percepción de carga o burden, como ha quedado demostrado a lo 
largo de la presente revisión bibliógrafica. En palabras de Menezes de Lucena Cavalho et al. (2006), aunque en referencia a cuidadores profesionales, "no se puede concluir que los cuidadores más resilientes no llegan a quemarse (Burnout), sino que logran mayores habilidades y competencias de compromiso laboral (vigor, dedicación y absorción), y en la medida que refuerzan estos atributos personales, mitigan la vulnerabilidad al Burnout" (Menezes Lucena Cavalho et al., 2006, p. 791).

\section{Conclusiones generales}

Lo primero que se observa al analizar la producción científica acerca de la resiliencia (y la personalidad resistente) en cuidadores no profesionales de mayores con demencia es que, como ya se ha señalado reiteradamente, no se dispone por el momento de una línea de investigación lo suficientemente clara y delimitada como para conciliar los resultados de una manera fiable. Cada autor adopta una definición diferente para estos conceptos, y las medidas empleadas para su evaluación difieren considerablemente entre estudios. A este problema, además, se suma la escasez de artículos publicados al respecto. No obstante, y a pesar de las muchas limitaciones a las que nos enfrentamos, los datos disponibles hasta la fecha nos permiten extraer diversas conclusiones significativas.

En primer lugar, aunque los cuidadores se hallan sometidos a un alto grado de estrés, en más ocasiones de las que se espera dan cuenta de elevados niveles de resiliencia y de hardiness, lo cual disminuye la probabilidad de desarrollar mucha de la sintomatología psicológica asociada a situaciones tan altamente demandantes. Además, un gran número de cuidadores aprecian un crecimiento personal a través de la experiencia de asistencia.

Tanto la resiliencia como la personalidad resistente del cuidador se hallan vinculadas a un conjunto de factores que interactúan, entre los que destacan los relacionados con la forma de evaluación, el tipo de afrontamiento llevado a cabo por el sujeto, y el apoyo social recibido. No obstante, y a pesar de que la correlación entre estos factores es sin duda alguna significativa, el modo en que se comporta cada uno aún está por descubrir.
Un modelo hipotético podría dibujar la interacción de estas variables de la siguiente manera: las personas resilientes evaluarán los estresores derivados del cuidado en términos positivos (o menos negativos) que las no resilientes, lo cual las conducirá en muchos casos a emplear estilos de afrontamiento eficaces y a evitar decisiones que surjan de una excesiva focalización en la emoción. Evaluaciones en términos de compromiso, desafío y control constituyen una manera eficaz para lograr estos resultados. Además, el apoyo social disponible presumiblemente servirá para apuntalar la resiliencia del cuidador, amortiguando las demandas del cuidado. Es decir, el apoyo social, en cuidadores, además de influir directamente en un buen número de emociones positivas, se asocia a la provisión de ayuda, lo cual contribuirá a aligerar en mayor o menor medida la carga objetiva y subjetiva que resulta del cuidado. Una menor sensación de carga ayudará, asimismo, a salvaguardar la resiliencia del cuidador.

Otra interpretación, sin embargo, también es plausible. Si bien las personas resilientes tienden a evaluar y a afrontar eficazmente las situaciones de estrés que atraviesan, una evaluación positiva y un estilo de afrontamiento eficaz tendrán una mayor probabilidad de generar consecuencias deseables. Cuidadores que afronten con éxito la experiencia de cuidado verán atenuado el efecto de muchos de los estresores primarios y secundarios que la conforman, por lo que su nivel de resiliencia se verá incrementado. Además los cuidadores resilientes tenderán a valorar con mayor optimismo el apoyo social que reciben y, al mantener un funcionamiento estable, es posible que sean capaces de generar y proteger con mayor éxito sus relaciones sociales. Otras variables (e.g. variables sociodemográficas) también han de ser contempladas en los modelos de resiliencia.

En conjunto, tanto el hardiness como la resiliencia se asocian a diferentes factores, cuyas relaciones todavían están por descubrir, si bien parece que un modelo integrado debería contemplar influencias de tipo bidireccional. Esperamos que en el futuro nuevos estudios permitan despejar todos los interrogantes que ensombrecen ambos conceptos, y se consoliden los cuidadores no profesionales de mayores dependientes como una de las principales poblaciones de referencia. 
Por último, no hay que olvidar que el mantenimiento de la situación de cuidado a lo largo del tiempo puede presentarse como una ocasión para el desarrollo de experiencias de crecimiento personal, extrayendo muchos cuidadores un valioso aprendizaje de su lucha contra la adversidad y viendo, así, reforzado su autoconcepto. Inter- venciones dirigidas a promover tales experiencias deben constituir siempre un complemento a los tradicionales tratamientos centrados en la sintomatología. En definitiva, el cuidado de un ser querido, con los sacrificios que ello conlleva, encierra siempre una decisión cargada de sentido y generosidad.

\section{Extended Summary}

\section{Objectives of present work}

The care of elderly people with dementia by their relatives is a very topical problem. Increasing numbers of studies show that non-professional caregivers are subject to high levels of stress, and that many of them develop psychological symptoms (e.g. depression, anxiety, anger or burden). However, recent years, different researchers have emphasized that caregiving for dependent elderly people is also an important source of personal satisfaction and growth. From this point of view it make sense to test caregiver's resilience and positive consequences.

The current article presents a systematical review of those studies that assess resilience, hardiness and personal growth in non-professional caregivers of dementia patients. The analysis of this kind of research is important for several reasons. From a theoretical point of view, it is necessary to study resilience, and related concepts, in the framework of chronic stressors, since resilience is almost always considered in relation to life or traumatic events and posttraumatic stress.

Furthermore, the recent available data are dispersed and heterogeneous. For this reason, the systematic review of the current results and findings would be of interest as a first step to advance research on this topic. Additionally, and from a clinical perspective, identifying which factors make some caregivers more resilient than others is a critical issue for the development of programs focused on prevention of the negative effects associated with non-professional care.

\section{Methodology}

The Medline and Psycinfo databases were con- sulted, with 'resilience', 'growth' and 'hardiness' introduced as key words, and by checking their combination with the concepts of 'care', 'caregiving' and 'caregiver', the works were selected that focused on the non-professional care of elderly people with dementia. All articles that considered other care recipient populations were discarded.

All in all, nine studies were selected that assess resilience, hardiness or growth in family caregivers of dementia patients; seven of them had been carried out in the United States, one in Germany and one in Canada. All the selected articles used a co-relational method with regression procedures for the analysis of results.

\section{Results}

The nine studies note the presence of resilience, hardiness and growth in relatives of dementia patients. Moreover, resilience was compatible with the existence of various levels of psychological symptoms. Therefore, a caregiver, may score highly in resilience as well as in depression or anxiety, for example, showing that the positive and negative effects of caregiving are not mutually independent.

In addition, the available data show that both resilience and hardiness are associated to several factors, which, according to Gaugler et al. (2007), could be classified into the following categories: (a) context of care (age, kin, relationship, duration of care, gender, and socioeconomic status), (b) care recipient status (cognitive impairment, unmet care needs, and primary caregiving hours), and (c) individual, family, and community resources (secondary support, community-based services, caregiver function, and intrapsychic resources). The caregiver's resilience would interact with these variables, and 
could determine the transitions in the dementia caregiving process (i.e. institutionalization, care recipient death, and loss at follow-up).

Specifically, the results show that both resilience and hardiness depend on variables such as social support or elderly status. However, it seems that the intrapychic resources of caregivers are more strongly associated to the caregiver's resilience. Therefore caregiver's appraisals and coping are key issues to the development of resilience: resilient caregivers tend to use problem-focused coping, and they make more positive appraisals than non-resilient caregivers. For Braithwaite (2000) the resiliency appraisal of a caregiver reflects personal resources such high self-esteem, mastery, good physical health, and emocional stability; and the conjunction of these resources results in inner strength and efficacy.

Similarly, hardiness is predominantly associated to effective coping (i.e. transformational coping strategies or willingness to seek help) and caregiver's appraisals. Indeed, hardiness is often conceived as a personality macrofactor (composed of the commitment, control and change dimensions) that helps the development of resilience, and consequently both hardiness and resilience would be influenced by similar factors.

On the other hand, though resilience is not incompatible with psychological symptoms, high scores in resilience would predict low scores in depression, burden or anxiety. In fact, the strictest definitions of resilience require the absence of symptoms.

Finally, many caregivers, besides showing resilience, relate experiences of personal growth due to the care situation. These experiences appear to be linked to demanding situations, in which the caregiver develops coping strategies such as coping by reframing or coping with religion. Additionally, social support was associated with the tendency to show personal growth experiences.

\section{Conclusions}

The main problem when analyzing resilience, hardiness and growth in caregivers of dementia patients is the lack of an integrative model. Each researcher employs different definitions for these concepts and, therefore, it is difficult to reconcile findings. Nevertheless, the resilience study (hardiness and personal growth) show interesting results.

Firstly, although caregivers suffer high stress levels, they often obtained high scores on resilience and hardiness. This caregiver's resistance depends of numerous factors, particularly individual or personal ones. In this sense, the coping strategies and caregiver appraisals seem to be the most important variables to caregiver's resilience. Despite this, the way in which these factors interact with caregiver's resilience is yet to be clarified. That is to say that, a resilient (or hardy) person, for example, could use effective coping strategies, and would obtain optimal results in their adaptation to circumstances. But it is also possible that the use of effective coping strategies helps caregivers to increase their resilience. Coping could therefore be considered both a cause or a consequence of caregiver's resilience.

Secondly, it is known that resilient caregivers will probably be less depressed and less burdened than non-resilient ones. Resilience is therefore a consolidated predictor for good personal results. Hence the importance of the study of resilience in the caregiving context.

Finally, many caregivers, in addition to successfully coping with a caregiving situation, come to find, in such a stressful experience, an opportunity for personal growth. The care of a loved one can be a very rewarding experience and, for this reason, any psychological intervention aimed to treat caregiver's burden should also promote learning and personal growth.

\section{Referencias}

Baker, K., y Robertson, N. (2008). Coping with caring for someone with dementia: Reviewing the literature about men. Aging \& Mental Health, 12, 413-422.

Bartone, P. (1991, Junio). Development and validation of a short hardiness measure. Presentado en la tercera convención anual de la American Psychological Society. Washington, DC. 
Becoña, E. (2006). Resiliencia: definición, características y utilidad del concepto. Revista de Psicopatología y Psicología Clínica, 11, 125-146.

Bergeman, C., y Wallace, K. (1999). Resiliency in later life. En T. Whitman, Merluzzi, y R. White, Life-span perspectives on health and illness ( $\mathrm{pp}$. 207-225). Mahwah, Nueva York: Erlbaum.

Block, J., y Kremen, A. (1996). IQ and EgoResiliency: Conceptual and empirical connections and separateness. Journal of Personality and Social Psychology, 70, 349-361.

Bonanno, G. (2004). Loss, trauma, and human resilience: Have we underestimated the human capacity to thrive after extremely aversive events? American Psychologist, 59, 20-28.

Braithwaite, V. (2000). Contextual or general stress outcomes: Making choices through caregiving appraisals. The Gerontologist, 40, 706-717.

Calhoun, L., y Tedeschi, R. (2000). Early Posttraumatic Interventions: Facilitating Posibilities for Growth. En J. Violanti, D. Patton, y D. Dunning. Posttraumatic Stress Intervention: Challenges, Issues and Perspectives (pp. 135152). Springfield, IL,USA: Charles C. Thomas Publisher.

Clark, L., y Hartman, M. (1996). Effects of hardiness and appraisal on the psychological distress and physical health of caregivers to ederly relatives. Research on Aging, 18, 379-401.

Clark, P. (2002). Effects of individual and family hardiness on caregiver depression and fatigue. Research in Nursing \& Health, 25, 37-48.

Cohen, C., Colantonio, A., y Vernich, L. (2002). Positive aspects of caregiving: Rounding out the caregiver experience. International Journal of Geriatric Psychiatry, 17, 184-188.

Connor, K., y Davidson, J. (2003). Development of a new resilience scale: The Connor-Davidson Resilience Scale (CD-RISC). Depression and Anxiety, 18, 76-82.

DiBartolo, M., y Soeken, K. (2003). Appraisal, coping, hardiness, and self-perceived health in community-dwelling spouse caregivers of persons with dementia. Research in Nursing \& Health, 26, 445-458.

Gaugler, J., Kane, R., y Newcomer, R. (2007). Resilience and transitions from dementia caregiv- ing. The Journals of Gerontology: Psychological Sciences, 62 B, 38-44.

Goode, K. T., Haley, W. E., Roth, D., y Ford, R. (1998). Predicting longitudinal changes in caregiver physical and mental health: A stress process model. Health Psychology, 17, 190-198.

Hardiness Institute (1985). Personal Views Survey. Arlington Heights, Illinois: Author

Heru, A., y Ryan, C. (2006). Family functioning in the caregivers of patients with dementia: Oneyear follow-up. Bulletin of the Menninger Clinic, 70, 222-231.

Hogan, N., Greenfield, D., Schmidt, L. (2001). Development and validation of the Hogan Grief Reaction Checklist. Death Studies, 25, 1-32.

IMSERSO (2005). Cuidados a las personas mayores en los hogares españoles. El entorno familiar. Madrid: IMSERSO.

Instituto Nacional de Estadística (2008). Encuesta de Discapacidad, Autonomía personal y situaciones de Dependencia (EDAD) Madrid: INE.

Katz, S., Ford, A., Moskowitz, R., Jackson, B, y Jaffe, M. (1963). Studies of illness in the aged. The index of ADL: A standardized measure of biological and psychosocial function. Journal of the American Medical Association, 185, 914-919.

Kinney, J., y Parris Stephens, M. (1989). Hassles and uplifts of giving care to a family member with dementia. Psychology and Aging, 4, 402408.

Kobasa C. (1979). Stressful life events, personality, and health: An inquiry into hardiness. Journal of Personality and Social Psychology, 37, 1-11.

Kramer, B. (1997). Differential predictors of strain and gain among husbands caring for wives with dementia. The Gerontologist, 37, 239-249.

Lawton, M., y Brody, E. (1969). Assessment of older people: Self-maintaining and instrumental activities of daily living. The Gerontologist, 9, 179-186.

Lazarus, R., y Folkman, S. (1984). Stress, appraisal, and coping. New York: Springer.

Leipold, B., Schacke, C., y Zank, S. (2008). Personal growth and cognitive complexity in caregivers of patients with dementia. European Journal of Ageing, 5, 203-214.

Liming Alspaugh, M., Zarit, S., y Greene, R. (1999). 
Longitudinal patterns of risk for depression in dementia caregivers: Objective and subjective primary stress as predictors. Psychology and Aging, 14, 34-43.

Luthar, S., Cicchetti, D., y Becker, B. (2000). The construct of resilience. A critical evaluation and guidelines for future work. Child Development, 71, 543-562.

McCubbin, M., McCubbin, H., y Thompson, A. I. (1986). Family Hardiness Index (FHI). En H. McCubbin, A. Thompson, y M. McCubbin (1996), Family assessment: Resiliency, coping and adaptation-Inventories for research and practice (pp. 239-305). Madison, WI: University of Wisconsin-Madison.

Maddi, S. (2005). On hardiness and other pathways to resilience. American Psychologist, 60, 261262.

Maddi, S. (2008). The courage and strategies of hardiness as helpful in growing despite major, disruptive stresses. American Psychologist, 63, 563564.

Masten, A. (2001). Ordinary Magic: Resilience processes in develpment. American Psychologist, 56, 227-238.

Menezes de Lucena Cavalho, V., Fernández Calvo, B., Hernández Martín, L., Ramos Campos, F., y Contador Castillo, I. (2006). Resiliencia y el modelo de Burnout-Engagement en cuidadores formales de ancianos. Psicothema, 18, 791-796.

Milne, C., Sacco, C., Cetinski, G., Browne, G., y Roberts, J. (1994). Correlates of well-being among caregivers of cognitively impaired relatives. Canadian Journal of Nursing Research, 26, 27-39.

Neill, J.y Dias, K. (2001). Adventure education and resilience: A double-edged sword. Journal of Adventure Education and Outdoor Leadership, 1, 35-42.

Olsson, C., Bond, L., Burns, J., Vella-Brodorick, D., y Sawyer, S. (2003). Adolescent resilience: a concept anlysis. Journal of Adolescence, 26, 1-11.

Ott, C., Sanders, S., y Kelber, S. (2007). Grief and personal growth experience of spouses and adultchild caregivers of individuals with Alzheimer's disease and related dementias. The Gerontologist, 47, 798-809.
Pakenham, K., Chiu, J., Burnsall, S., y Cannon, T. (2007). Relations between social support, appraisal and coping and both positive and negative outcomes in young carers. Journal of Health Psychology, 12, 89-103.

Peñacoba, C., y Moreno, B. (1998). El concepto de personalidad resistente. Consideraciones teóricas y repercusiones prácticas. Boletín de Psicología, 58, 61-96.

Phelps, K., McCammon, S.,Wuensch, K., y Golden, J. (2009). Enrichment, stress, and growth from parenting an individual with an autism spectrum disorder. Journal of Intellectual and Developmental Disability, 34, 133-141.

Pinquart, M., y Sörensen, S. (2003a). Differences between caregivers and noncaregivers in psychological health and physical health: A meta-analysis. Psychology and Aging, 18, 250-267.

Pinquart, M., y Sörensen, S. (2003b). Associations of stressors and uplifts of caregiving with caregiver burden and depressive mood: A meta-analysis. The Journals of Gerontology: Series B: Psychological Sciences and Social Sciences, 58B, 112-128.

Pollock, S. (1989). The hardiness characteristic: A motivating factor in adaptation. ANS: Advances in Nursing Science, 11, 53-62.

Rapp, S., Shumaker, S., Schmidt, S., Naughton, M., y Anderson, R. (1998). Social resourcefulness: Its relationship to social support and wellbeing among caregivers of dementia victims. Aging \& Mental Health, 2, 40-48.

Ryff, C. (1989) Happiness is everything, or is it? Explorations on the meaning of psychological well-being. Journal of Personality and Social Psychology, 57, 1069-1081

Schulz, R., O'Brien, A., Bookwala, M., y Fleissner, K. (1995). Psychiatric and physical morbidity effects of dementia caregiving: Prevalence, correlates, and causes. Gerontologist, 35, 771-791.

Tedeschi, R., y Calhoun, L. (2004). Target Article: "Posttraumatic Growth: Conceptual Foundations and Empirical Evidence". Psychological Inquiry, 15, 1-18.

Tedeschi, R., y Kilmer, R. (2005). Assessing strengths, resilience, and growth to guide clinical interventions. Professional Psychology: Research 
and Practice, 36, 230-237.

Townsend, A., Noelker, L., Deimling, G., y Bass, D. (1989). Longitudinal impact of interhousehold caregiving on adult children's mental health. Psychology and Aging, 4, 393-401.

Vitaliano, P., Schulz, R., Kiecolt-Glaser, J., y Grant, I. (1997). Research on physiological and physical concomitants of caregiving. Where do we go from here? Annals of Behavior Medicine, 19, 117123.

Wagnild, G., y Young, H. (1993). Development and psychometric evaluation of the resilience scale. Journal of Nursing Measurement, 1, 165-178.

Wilks, S., y Croom, B. (2008). Perceived stress and resilience in Alzheimer's disease caregivers:
Testing moderation and mediation models of social support. Aging \& Mental Health, 12, 357365.

Wilks, S., y Vonk, M. (2008). Private prayer among Alzheimer's caregivers: mediating burden and resiliency. Journal of Gerontological Social Work, 50, $113-131$.

Zarit, S., Orr, N., y Zarit, J. (1985). Understanding the stress of caregivers: Planning and intervention. En S. Zarit, N. Orr, y J. Zarit, Hidden victims of Alzheimer's disease: Families under stress (pp. 69-86). New York: NYU Press.

Zarit, S., Todd, P., y Zarit, J. (1986). Subjective burden of husbands as wives as caregivers: A longitudinal study. The Gerontologist, 26, 260-266.

Manuscrito recibido: $15 / 03 / 2010$

Revisión recibida: 14/07/2010

Manuscrito aceptado: 08/10/2010 